\title{
Distributional Sources in General Relativity: two point-like examples revisited
}

\author{
N. R. PANTOJA \\ Centro de Astrofísica Teórica. \\ Departamento de Física, Facultad de Ciencias. Universidad de los Andes. \\ Mérida, 5101, Venezuela. \\ H. RAGO \\ Laboratorio de Física Teórica and Centro de Astrofísica Teórica. \\ Departamento de Física, Facultad de Ciencias. Universidad de los Andes. \\ Mérida, 5101, Venezuela.
}

\begin{abstract}
A regularization procedure, that allows one to relate singularities of curvature to those of the Einstein tensor without some of the shortcomings of previous approaches, is proposed. This regularization is obtained by requiring that (i) the density $|\operatorname{det}|^{\frac{1}{2}} G_{b}^{a}$, associated to the Einstein tensor $G_{b}^{a}$ of the regularized metric, rather than the Einstein tensor itself, be a distribution and (ii) the regularized metric be a continuous metric with a discontinuous extrinsic curvature across a non-null hypersurface of codimension one. In this paper, the curvature and Einstein tensors of the geometries associated to point sources in the $2+1$-dimensional gravity and the Schwarzschild spacetime are considered. In both examples the regularized metrics are continuous regular metrics, as defined by Geroch and Traschen, with well defined distributional curvature tensors at all the intermediate steps of the calculation. The limit in which the support of these curvature tensors tends to the singular region of the original spacetime is studied and the results are contrasted with the ones obtained in previous works.
\end{abstract}

\section{INTRODUCTION}

Within the framework of general relativity, a spacetime singularity corresponds to a singularity of the metric tensor which can not be removed by a coordinate transformation, even one which itself becomes singular where the metric does. On the other hand, it is expected that this singularity reveals itself through a lack of smoothness of the curvature tensor. In order to incorporate singular curvatures into general relativity, distributional curvatures have been considered in several papers [1]. However, as is well known, computing the curvature tensor from a metric requires nonlinear operations which are not defined within the framework of distribution theory. This imposes strong constraints on the class of metrics whose curvature tensors make sense as distributions.

There is a class of metrics, the regular metrics [4], for which the curvature tensor has a well defined distributional meaning. The singular parts of the curvature tensor of these regular metrics for a $d$-dimensional spacetime are supported on submanifolds of codimension of at most one, i.e., of dimension $\geq d-1$, and for these metrics it makes sense to write Einstein's equations with energy-momentum tensor distributions. Metrics for surface layers [5], turn out to be included into the class of regular metrics [4.66 8]. However, there are some very simple metrics, from which a physically interesting spacetime follows, which are not regular. One example is the $3+1$-dimensional Minkowski metric with an angular deficit [4], 9], another is the Schwarzschild metric [4]. Regular metrics are a subclass of a larger class of metrics, semi-regular metrics [9]. The curvature of a semi-regular metric is well defined as a distribution and it has been proved that the $3+1$-dimensional Minkowski metric with an angular deficit and a certain kind of traveling wave metric are semi-regular metrics [9]. Alternatively, by considering the Colombeau's theory of generalized functions [10], distributional curvatures can be defined. This approach has been used to obtain the distributional curvature associated to a conical singularity $111-13$ but we will not consider it here.

By invoking regularization procedures, a distributional meaning may be given to the curvature tensor of a nonregular metric. However, the reference differentiable structure that the regularization implicitly uses, makes uncertain the independence of the result on the regularization procedure chosen [14]. It is well known that problems appear in defining the curvature tensor for the $3+1$-dimensional Minkowski spacetime with an angular deficit, due to the fact that the distributional limit depends on the choice of regularization [4]. Regularizations of the metric have been also used to calculate distributional curvatures for the Schwarzschild [15,16] and Kerr-Newman spacetimes [17], with results which are regularization scheme dependent 16.

In this work we propose a very restrictive kind of regularization inspired by the approach used in early works to the study of the classical gravitational self-energy and the minimum extension associated to point-like sources in general 
relativity 18,19$]$. We show that this approach may be used to regularize non-regular metrics in such a way that the regularized metrics are continuous regular metrics with well defined distributional curvature tensors, in the sense of reference [4], at every intermediate step in the calculation. Furthermore, since continuous regular metrics can be suitably approximated by smooth metrics 4 , this approach provides a physically sensible idealization for the smooth metrics of general relativity.

In section II, after an overview on the subject of distributions on metric manifolds, we review the definitions of regular and semi-regular metrics. In the following section, the distributional curvature and Einstein tensors of the $(2+1)$-dimensional spacetime around a massive point source [20] are considered in some detail. First, following Ref. [9], we show that the metric of this spacetime is a semi-regular metric with a well defined distributional curvature but with an Einstein tensor which is zero everywhere. Next, we regularize the metric by requiring that (i) the tensor density $|\operatorname{det} \mathbf{g}|^{\frac{1}{2}} G_{b}^{a}$ associated to the mixed-index Einstein tensor $G_{b}^{a}$, rather than the Einstein tensor itself, be a well defined distribution and (ii) the regularized metric be a continuous metric with a discontinuous extrinsic curvature across a non-null hypersurface of codimension one. The distributional limit to a point source is shown to be in agreement with the identification made in reference [20]. Finally, we show that the regularized metric is a regular metric and, following the approach of Ref. [4], calculate the curvature and Einstein tensors. The distributional limit in which the regularization is removed reveals the origin of the disagreement between the results obtained. In section IV, the Schwarzschild spacetime is considered. We first prove that the Schwarzschild metric is not a semi-regular metric and show some drawbacks of previous regularization approaches. Next, following the regularization procedure of section IIII, we regularize the Schwarzschild metric obtaining a regular metric. The results, in the limit in which the regularization is removed, are shown to be in agreement with those obtained in previous works. Finally, following the approach of Ref. [4], we calculate the curvature and Einstein tensors of the regularized metric. The results obtained by taking the limit in which the regularization is removed are then contrasted with the previous ones.

\section{REGULAR AND SEMI-REGULAR METRICS}

Let us briefly review the class of metrics which have been defined as regular and semi-regular metrics. For this, we first recall some fundamental results about distributions. Since we shall have no need for the theory of distributions on arbitrary manifolds in its greatest generality [21,22], we shall simplify wherever possible.

Let $\Phi(\mathcal{M})$ be the family of $C^{\infty}$ real-valued scalar functions $\phi$ defined on an orientable $n$-dimensional $C^{\infty}$ paracompact manifold $\mathcal{M}$ and vanishing outside some compact region of $\mathcal{M}$. Further, suppose that a rule can be introduced in $\Phi(\mathcal{M})$ defining the convergence to zero of a sequence of functions $\phi_{n}(n=1,2, \ldots)$ belonging to $\Phi(\mathcal{M})$.

Let $\mathbf{g}$ be a metric on $\mathcal{M}$ with associated volume element $\omega_{\mathbf{g}}=|\operatorname{det} \mathbf{g}|^{\frac{1}{2}} \varepsilon$, where $\varepsilon=\mathbf{d} x^{1} \wedge \ldots \wedge \mathbf{d} x^{n}$ is the associated coordinate volume element. We can define the functional generated by the 0 -form $f$ on $\Phi(\mathcal{M})$ through the rule

$$
\int_{\mathcal{M}} \omega_{\mathbf{g}} f \phi=\int_{U_{\mathcal{M}}} d x^{1} \ldots d x^{n}|\operatorname{det} \mathbf{g}|^{\frac{1}{2}} f(x) \phi(x),
$$

where $U_{\mathcal{M}}$ is the coordinate domain corresponding to $\mathcal{M}$. Obviously, this identification does not depend on the choice of coordinate system covering the corresponding domain, but depends on the choice of $\mathbf{g}$ through the volume element $\omega_{\mathrm{g}}$.

Let $\eta$ be a $C^{\infty}$ metric tensor. If we endow the manifold $\mathcal{M}$ with such a metric, every locally integrable 0 -form $f$ defines a distribution through (11), and write

$$
f[\phi]=\int_{\mathcal{M}} \omega_{\eta} f \phi
$$

However, it should be noted that Eq.(1) allows one to consider the functional generated by the scalar density $|\operatorname{det}|^{\frac{1}{2}} f$, even when $\mathbf{g}$ is a $C^{k}$ metric tensor.

The extension to tensor distributions is straightforward. Let $\mathbf{U}$ be a smooth $\left(\begin{array}{l}q \\ p\end{array}\right)$-tensor with compact support on $\mathcal{M}$. A locally integrable $\left(\begin{array}{l}\mathrm{p} \\ \mathrm{q}\end{array}\right)$-tensor field $\mathbf{T}$ is identified with a tensor distribution via

$$
\mathbf{T}[\mathbf{U}] \equiv \int_{\mathcal{M}}(\mathbf{T} \mid \mathbf{U}) \omega_{\eta},
$$

where

$$
(\mathbf{T} \mid \mathbf{U}) \equiv T^{i_{1} \ldots i_{p} \ldots j_{q}} U^{j_{1} \ldots j_{i_{1}} \ldots i_{p}} .
$$


A ( $\left(\begin{array}{l}\mathrm{p} \\ \mathrm{q}\end{array}\right)$-tensor field $\mathbf{T}$ is called locally bounded provided that (何) is bounded for all test $\left(\begin{array}{c}\mathrm{q} \\ \mathrm{p}\end{array}\right)$-tensor fields $\mathbf{U}$. The derivative in the smooth metric $\eta$ of a $\left(\begin{array}{l}p \\ q\end{array}\right)$-tensor distribution $\mathbf{T}$ is the $\left(\begin{array}{c}p \\ q+1\end{array}\right)$-tensor defined for every test $\left(\begin{array}{c}q+1 \\ p\end{array}\right)$-tensor $\mathbf{U}$ by

$$
\nabla \mathbf{T}[\mathbf{U}]=-\mathbf{T}[\eta \cdot \nabla \mathbf{U}]
$$

where

$$
(\eta \cdot \nabla \mathbf{U})^{j_{1} \ldots j_{q_{i}} \ldots i_{p}}=\nabla_{j} U^{j j_{1} \ldots j_{q_{1}} \ldots i_{p}} .
$$

Finally, the weak derivative of a locally integrable $\left(\begin{array}{c}p \\ q\end{array}\right)$-tensor tensor field $\mathbf{T}$ is a locally integrable $\left(\begin{array}{c}\mathrm{p} \\ \mathrm{q}+1\end{array}\right)$-tensor field $\mathbf{W}$, if one exist, such that

$$
\mathbf{W}[\mathbf{U}]=\nabla \mathbf{T}[\mathbf{U}]
$$

for every test $\left(\begin{array}{c}q+1 \\ p\end{array}\right)$-tensor field $\mathbf{U}$. For an approach in which tensor distributions and their derivatives are described without assuming the presence of a metric see reference [23]. Alternatively, by using de Rham currents [24] and replacing test tensors by test $n$-forms, the introduction of a volume element can be avoided. Here we shall follow the more conventional approach of reference [4].

Now, let $\nabla$ be the derivative operator in any smooth metric $\eta$. Then the Riemann tensor of a smooth metric $\mathbf{g}$ can be written as

$$
R_{a b c}^{d}=\tilde{R}_{a b c}^{d}+2 \nabla_{[b} C_{a] c}^{d}+2 C_{m[b}^{d} C_{a] c}^{m}
$$

where

$$
\left.C_{a b}^{c} \equiv \frac{1}{2}\left(g^{-1}\right)^{c d}\left(\nabla_{a} g_{b d}+\nabla_{b} g_{a d}-\nabla_{d} g_{a b}\right)\right)
$$

and $\tilde{R}_{a b c}{ }^{d}$ is the curvature tensor of $\eta$. For the Einstein tensor of $\mathbf{g}$ we have

$$
G_{a b}=R_{a b}-\frac{1}{2}\left(g^{-1}\right)^{c d} \tilde{R}_{c d} g_{a b}+\left(g^{-1}\right)^{c d} C_{m[c}^{e} C_{e] d}^{m} g_{a b}+\nabla_{[c}\left(C_{e] d}^{e}\left(g^{-1}\right)^{c d} g_{a b}\right)+C_{d[c}^{e} \nabla_{e]}\left(\left(g^{-1}\right)^{c d} g_{a b}\right),
$$

where $R_{a b}=R_{a c b}^{c}$ is the Ricci tensor.

Following Ref. 4 , a symmetric tensor field $\mathbf{g}$ on a manifold $\mathcal{M}$ will be called a regular metric provided: (i) $\mathbf{g}$ and $\mathbf{g}^{-1}$ exist everywhere and are locally bounded, and (ii) the weak derivative of $\mathbf{g}$ in some smooth metric $\eta$ exists and is locally square-integrable, i.e., the outer product of the weak derivative with itself is locally integrable. The curvature tensor (8) and the Einstein tensor (10) of a regular metric make sense as distributions, therefore it makes sense to write Einstein's equations with distributional energy-momentum tensors. It turns out that these idealized matter sources must be concentrated on submanifolds of codimension of at most one 四.

A wider class of metrics whose curvature makes sense as a distribution can be defined, the so-called semi-regular metrics [9]. A symmetric tensor field $\mathbf{g}$ on a manifold $\mathcal{M}$ will be called a semi-regular metric provided: (i) $\mathbf{g}$ and $\mathbf{g}^{-1}$ exist almost everywhere and are locally integrable, and (ii) the weak first derivative $\nabla \mathbf{g}$ of $\mathbf{g}$ in some smooth metric $\eta$ exist and the tensors $C_{a b}^{c}$ and $C_{m[b}^{d} C_{a] c}^{m}$ are locally integrable. Under these constraints, the right hand side of (8) is well defined as a distribution. However, a semi-regular metric may have no distributional Einstein tensor. This is due to the fact that for a semi-regular metric, contractions of the metric with the curvature tensor may have no sense as distributions.

\section{POINT SOURCES IN $(2+1)$-DIMENSIONAL GRAVITY}

Consider a $(2+1)$-dimensional spacetime $\left(\mathcal{R}^{3}, \mathbf{g}\right)$, where the metric $\mathbf{g}$ in a particular coordinate system is given by

$$
\mathbf{g}=-\mathbf{d} t \otimes \mathbf{d} t+\rho^{-8 m}\left(\mathbf{d} \rho \otimes \mathbf{d} \rho+\rho^{2} \mathbf{d} \varphi \otimes \mathbf{d} \varphi\right),
$$

where $-\infty<t<\infty, 0<\rho$ and $-\pi \leq \varphi \leq \pi$, with the surfaces $\varphi=-\pi, \pi$ identified. In Ref. [20], the metric (11) has been identified with the metric generated by a point source of mass $m$ at the origin. 
It is easy to see that (11) is not a regular metric (the analogous treatment of the problem in $(3+1)$-dimensional gravity has been considered elsewhere [4, 9]). Let $\eta$ be the ordinary Minkowski metric on $\mathcal{R}^{3}$ given by

$$
\eta=-\mathbf{d} t \otimes \mathbf{d} t+\mathbf{d} \rho \otimes \mathbf{d} \rho+\rho^{2} \mathbf{d} \varphi \otimes \mathbf{d} \varphi,
$$

and we take for the differentiable structure that in which $t, x=\rho \cos \varphi$ and $y=\rho \sin \varphi$ form a smooth chart. It follows that

$$
\mathbf{g}=\eta-\left(1-\rho^{-8 m}\right)\left(\mathbf{d} \rho \otimes \mathbf{d} \rho+\rho^{2} \mathbf{d} \varphi \otimes \mathbf{d} \varphi\right)
$$

and

$$
\mathbf{g}^{-1}=\eta^{-1}-\left(1-\rho^{8 m}\right)\left(\partial_{\rho} \otimes \partial_{\rho}+\rho^{-2} \partial_{\varphi} \otimes \partial_{\varphi}\right)
$$

We have that $\mathbf{g}$ and $\mathbf{g}^{-1}$ exist almost everywhere. Let $\mathbf{U}$ be a test $\left(\begin{array}{l}2 \\ 0\end{array}\right)$-tensor field on $\mathcal{R}^{3}$. We have

$$
\mathbf{g}[\mathbf{U}]=\int_{\mathcal{R}^{3}}(\eta \mid \mathbf{U}) \omega_{\eta}-\int_{\mathcal{R}^{3}}\left(1-\rho^{-8 m}\right)\left(U^{x x}+U^{y y}\right) \omega_{\eta}
$$

Therefore, $\mathbf{g}$ is locally integrable for $0 \leq 8 m<2$ but not locally bounded. Next, let $\mathbf{S}$ be a test $\left(\begin{array}{c}0 \\ 2\end{array}\right)$-tensor field on $\mathcal{R}^{3}$. It follows that

$$
\mathbf{g}^{-1}[\mathbf{S}]=\int_{\mathcal{R}^{3}}\left(\mathbf{S} \mid \eta^{-1}\right) \omega_{\eta}-\int_{\mathcal{R}^{3}}\left(1-\rho^{8 m}\right)\left(S_{x x}+S_{y y}\right) \omega_{\eta}
$$

Hence, $\mathbf{g}^{-1}$ is locally bounded and locally integrable for all $m \geq 0$. Finally, let $\mathbf{U}$ be a test $\left(\begin{array}{c}3 \\ 0\end{array}\right)$-tensor field on $\mathcal{R}^{3}$. We find that the weak derivative in $\eta$ of $\mathbf{g}$ exist almost everywhere and is given by

$$
\nabla \mathbf{g}[\mathbf{U}]=-\mathbf{g}[\eta \cdot \nabla \mathbf{U}]=\lim _{\epsilon \rightarrow 0} \int_{\rho>\epsilon} g_{a b} \nabla_{c} U^{c a b} \omega_{\eta}=\int_{\mathcal{R}^{3}} W_{c a b} U^{c a b} \omega_{\eta}
$$

where

$$
W_{c a b}=-8 m \rho^{-(1+8 m)} d \rho_{c}\left(d \rho_{a} d \rho_{b}+\rho^{2} d \varphi_{a} d \varphi_{b}\right) .
$$

It then follows that $\nabla \mathbf{g}$ is not locally square integrable for $m>0$. Therefore, $\mathbf{g}$ is not a regular metric in the differentiable structure chosen. Actually, there exists no differentiable structure that will render the metric (11) regular since the support of the curvature of (11) is expected to be a submanifold of codimension greater than one.

Now, from (11) and (9) it follows that

$$
C_{a b}^{c}=\frac{4 m}{\rho}\left(\partial_{\rho}^{c} d \rho_{a} d \rho_{b}+\partial_{\varphi}^{c}\left(d \rho_{a} d \varphi_{b}+d \rho_{b} d \varphi_{a}\right)-\rho^{2} \partial_{\rho}^{c} d \varphi_{a} d \varphi_{b}\right),
$$

which is locally integrable. On the other hand

$$
C_{m[b}^{d} C_{a] c}^{m}=0,
$$

which is also locally integrable. Therefore, the metric (11) is a semi-regular metric.

From (8), (19) and (20), the Ricci tensor is given by

$$
R_{a b}=\nabla_{[c} C_{a] b}^{c} .
$$

Hence

$$
R_{a b}\left[S^{a b}\right]=-\int_{\mathcal{R}^{3}}\left(C_{a b}^{c} \nabla_{c} S^{a b}-C_{c b}^{c} \nabla_{a} S^{a b}\right) \omega_{\eta}
$$

and we obtain 


$$
\begin{aligned}
R_{a b}\left[S^{a b}\right] & =-\lim _{\epsilon \rightarrow 0} \int_{\rho>\epsilon}\left(C_{a b}^{c} \nabla_{c} S^{a b}-C_{c b}^{c} \nabla_{a} S^{a b}\right) \omega_{\eta} \\
& =\lim _{\epsilon \rightarrow 0}\left(\int_{\rho=\epsilon}\left(\nabla_{c} \rho C_{a b}^{c}-\nabla_{a} \rho C_{c b}^{c}\right) S^{a b} \sigma+\int_{\rho>\epsilon}\left(\nabla_{c} C_{a b}^{c}-\nabla_{a} C_{c b}^{c}\right) S^{a b} \omega_{\eta}\right),
\end{aligned}
$$

where $\sigma$ is the volume element induced on the surface $\rho=$ constant by the metric (12). For $\rho \neq 0$ we have

$$
\nabla_{c} C_{a b}^{c}=\nabla_{a} C_{c b}^{c}=\frac{8 m}{\rho^{2}}\left(d \rho_{a} d \rho_{b}-\rho^{2} d \varphi_{a} d \varphi_{b}\right)
$$

Then

$$
\begin{aligned}
R_{a b}\left[S^{a b}\right] & =-\lim _{\epsilon \rightarrow 0} \int_{\rho=\epsilon}\left(d \rho_{c} C_{a b}^{c}-d \rho_{a} C_{c b}^{c}\right) S^{a b} \sigma \\
& =\lim _{\epsilon \rightarrow 0} \int_{\rho=\epsilon} \frac{4 m}{\rho}\left(d \rho_{a} d \rho_{b}+\rho^{2} d \varphi_{a} d \varphi_{b}\right) S^{a b} \sigma \\
& =\lim _{\epsilon \rightarrow 0} 4 m \int_{\rho=\epsilon} d t d \varphi\left(d x_{a} d x_{b}+d y_{a} d y_{b}\right) S^{a b} \\
& =8 \pi m \int d t\left(S^{x x}(t, 0,0)+S^{y y}(t, 0,0)\right) .
\end{aligned}
$$

Therefore

$$
R_{a b}=8 \pi m \delta_{(0)}^{(2)}\left(d x_{a} d x_{b}+d y_{a} d y_{b}\right) .
$$

Next, from (10), (18) and (19), the Einstein tensor of (11) is given by

$$
G_{a b}=R_{a b}-8 m \nabla_{c}\left(-\rho^{8 m-1} \partial_{\rho}^{c} d t_{a} d t_{b}+\rho^{-1}\left(d \rho_{a} d \rho_{b}+\rho^{2} d \varphi_{a} d \varphi_{b}\right)\right)-64 m^{2} \rho^{8 m-2} d t_{a} d t_{b} .
$$

Note that the right hand side of (27) contains the derivative of a locally integrable tensor plus a locally integrable tensor. Hence, the Einstein tensor of (11) makes sense as a distribution. Then

$$
\begin{aligned}
G_{a b}\left[S^{a b}\right]= & R_{a b}\left[S^{a b}\right] \\
& +8 m \int_{\mathcal{R}^{3}}\left(-\rho^{8 m-1} \partial_{\rho}^{c} d t_{a} d t_{b}+\rho^{-1}\left(d \rho_{a} d \rho_{b}+\rho^{2} d \varphi_{a} d \varphi_{b}\right)\right) \nabla_{c} S^{a b} \omega_{\eta} \\
& -64 m^{2} \int_{\mathcal{R}^{3}} \rho^{8 m-2} d t_{a} d t_{b} S^{a b} \omega_{\eta} .
\end{aligned}
$$

An analogous calculation to that of (25) leads to

$$
\begin{aligned}
\int_{\mathcal{R}^{3}}\left(-\rho^{8 m-1} \partial_{\rho}^{c} d t_{a} d t_{b}\right. & \left.+\rho^{-1}\left(d \rho_{a} d \rho_{b}+\rho^{2} d \varphi_{a} d \varphi_{b}\right)\right) \nabla_{c} S^{a b} \omega_{\eta}= \\
& -\pi \delta_{(0)}^{(2)}\left(d x_{a} d x_{b}+d y_{a} d y_{b}\right)\left[S^{a b}\right]+8 m \int_{\mathcal{R}^{3}} \rho^{8 m-2} d t_{a} d t_{b} S^{a b} \omega_{\eta} .
\end{aligned}
$$

It follows from (26), (28) and (29) that

$$
G_{a b}=0 .
$$

These results suggest that the spacetime $\left(\mathcal{R}^{3}, \mathbf{g}\right)$ with $\mathbf{g}$ given by (11), although having a distributional curvature with support on the origin, has a zero everywhere distributional Einstein tensor. How are we to reconcile these results with those of Ref. [20]?

In the following, the approach of references 18,19] is considered. We show that this approach may be used to regularize (11) in such a way that the resulting regularized metric is a continuous regular metric with well defined distributional curvature and Einstein tensors in the sense of reference [4].

Consider a 3 -dimensional spacetime $\left(\mathcal{R}^{3}, \mathbf{g}\right)$, where the metric $\mathbf{g}$ is given by

$$
\mathbf{g}=g_{\alpha \beta} \mathbf{d} x^{\alpha} \otimes \mathbf{d} x^{\beta}=-\mathbf{d} t \otimes \mathbf{d} t+H\left(\mathbf{d} \rho \otimes \mathbf{d} \rho+\rho^{2} \mathbf{d} \varphi \otimes \mathbf{d} \varphi\right),
$$


where $-\infty<t<\infty, 0 \leq \rho$ and $-\pi \leq \varphi \leq \pi$, with the surfaces $\varphi=-\pi, \pi$ identified. At this stage, $H=H(\rho)$ is an unknown $C^{\infty}$ function, making possible to perform conventional pointwise differential geometry.

From (31), it follows that the curvature two-form, $\mathcal{R}_{\rho}^{\varphi}$, is given by

$$
\mathcal{R}_{\rho}^{\varphi}=\frac{1}{2} \frac{d}{d \rho}\left(\rho \frac{d}{d \rho} \log H\right) \mathbf{d} \rho \wedge \mathbf{d} \varphi
$$

and the non-zero components of the Riemann tensor are

$$
R_{\rho \varphi}^{\rho \varphi}=R_{\varphi \rho}^{\varphi \rho}=-\frac{1}{2 \rho H} \frac{d}{d \rho}\left(\rho \frac{d}{d \rho} \log H\right) .
$$

From (33), the Ricci tensor and the scalar curvature can be obtained. Finally, the Einstein tensor $\mathbf{G}$ is found to be given by

$$
\mathbf{G}=G_{t}^{t} \mathbf{d} t \otimes \partial_{t}
$$

where

$$
G_{t}^{t}=\frac{1}{2 \rho H} \frac{d}{d \rho}\left(\rho \frac{d}{d \rho} \log H\right)
$$

Here, it is worth recalling that in three spacetime dimensions the identity

$$
R_{\alpha \beta}^{\mu \nu}=\varepsilon^{\mu \nu \sigma} \varepsilon_{\alpha \beta \lambda} G_{\sigma}^{\lambda}
$$

holds, linking curvature and Einstein tensors.

Now, we take (34) as the definition of the Einstein tensor of (31). Let $\mathbf{U}$ be a test $\left(\begin{array}{l}1 \\ 1\end{array}\right)$-tensor field on $\mathcal{R}^{3}$. Next, consider the mixed-index Einstein tensor density $|\operatorname{det}|^{\frac{1}{2}} \mathbf{G}$, with $|\operatorname{det} \mathbf{g}|^{\frac{1}{2}}$ the density generated by the metric $\mathbf{g}$ in (31), as a functional on the space of test tensor fields through

$$
\int_{\mathcal{R}^{3}}|\operatorname{det} \mathbf{g}|^{\frac{1}{2}} G_{b}^{a} U_{a}^{b} \varepsilon=\int_{\mathcal{R}^{3}} d t d x d y H G_{t}^{t} U_{t}^{t}
$$

where it is understood that $G_{b}^{a}$ and $U_{a}^{b}$ are Cartesian components as functions of Cartesian coordinates $(t, x, y)$ with $x=\rho \cos \phi$ and $y=\rho \sin \phi$. Then

$$
\int_{\mathcal{R}^{3}}|\operatorname{det} \operatorname{g}|^{\frac{1}{2}} G_{b}^{a} U_{a}^{b} \varepsilon=\lim _{\epsilon \rightarrow 0} \int_{\rho>\epsilon} d t d \rho d \varphi \frac{1}{2} \frac{d}{d \rho}\left(\rho \frac{d}{d \rho} \log H\right) U_{t}^{t}(t, \rho \cos \varphi, \rho \sin \varphi)=\lim _{\epsilon \rightarrow 0} \pi \int_{\epsilon}^{\infty} d \rho \frac{d}{d \rho}\left(\rho \frac{d}{d \rho} \log H\right) \overline{U_{t}^{t}}(\rho),
$$

where

$$
\overline{U_{t}^{t}}(\rho) \equiv \frac{1}{2 \pi} \int_{-\infty}^{\infty} d t \int_{-\pi}^{\pi} d \varphi U_{t}^{t}(t, \rho \cos \varphi, \rho \sin \varphi)
$$

It follows that

$$
\int_{\mathcal{R}^{3}}|\operatorname{det} \mathbf{g}|^{\frac{1}{2}} G_{b}^{a} U_{a}^{b} \varepsilon=\lim _{\epsilon \rightarrow 0} \pi \int_{\epsilon}^{\infty} d \rho \log H \frac{d}{d \rho}\left(\rho \frac{d}{d \rho} \overline{U_{t}^{t}}\right)
$$

where we have integrated by parts and imposed the condition

$$
\lim _{\rho \rightarrow 0} \rho \frac{d}{d \rho} \log H=0 .
$$

Now, let us assume that (38) holds even for a less well-behaved $H$. Let $\log H$ be the $C^{1}$-function given by

$$
\log H=-8 m \log \rho_{>},
$$

where $\rho_{>}=\max \{\rho, \xi\}$ with $\xi$ a constant parameter which we will treat as a regulator. Eq.42 is a solution of 


$$
\frac{d}{d \rho}\left(\rho \frac{d}{d \rho} \log H\right)=-8 m \delta(\rho-\xi)
$$

that satisfies (41) and where both sides of (43) should be understood as distributions on test functions $\phi(\rho) \in \Phi\left(\mathcal{R}^{+}\right)$. From (42) it follows

$$
H(\rho)=\left(\rho_{>}\right)^{-8 m},
$$

which is also a well defined distribution on $\Phi\left(\mathcal{R}^{+}\right)$.

From (40) and (42) it follows

$$
\int_{\mathcal{R}^{3}}|\operatorname{det} \mathbf{g}|^{\frac{1}{2}} G_{b}^{a} U_{a}^{b} \varepsilon=-8 \pi m \overline{U_{t}^{t}}(\xi),
$$

where we have integrated by parts and used (41).

From (33), (36) and (45), we have that the Riemann tensor density is supported on the surface $\rho=\xi$. Hence, the spacetime will be flat except on this surface. Geometries of this kind in $(2+1)$-dimensional gravity have been previously studied 25,26$]$.

From (45), it follows

$$
\lim _{\xi \rightarrow 0} \int_{\mathcal{R}^{3}}|\operatorname{det} \mathbf{g}|^{\frac{1}{2}} G_{b}^{a} U_{a}^{b} \varepsilon=-8 \pi m \overline{U_{t}^{t}}(0)=-8 \pi m \int_{-\infty}^{\infty} d t U_{t}^{t}(t, 0,0) .
$$

Therefore, the $\xi \rightarrow 0$ limit of the density $|\operatorname{det} \mathbf{g}|^{\frac{1}{2}} \mathbf{G}$ is the distribution

$$
\lim _{\xi \rightarrow 0}|\operatorname{det}| \frac{1}{2} G_{b}^{a}=-8 \pi m \delta_{(0)}^{(2)} \partial_{t}^{a} d t_{b}
$$

where $\delta_{(0)}^{(2)}$ is the usual two-dimensional Euclidean $\delta$ distribution with support on the origin. This means that the spacetime $\left(\mathcal{R}^{3}, \mathbf{g}\right)$, where the metric $\mathbf{g}$ is given by $(31,44)$, can be identified in the $\xi \rightarrow 0$ limit with the spacetime generated by a distributional energy-momentum tensor $\mathbf{T}=T_{t}^{t} \mathbf{d} t \otimes \partial_{t}$ with

$$
|\operatorname{det} \mathbf{g}|^{\frac{1}{2}} T_{t}^{t}=-m \delta_{(0)}^{(2)} .
$$

Since for $\rho>\xi$, the metrics (11) and (31, 44 ) agree, a point source of mass $m$ at the origin may be considered as the source for the metric (11), as proposed in reference [20].

We now wish to make contact with the approach of reference 四. The regularized metric (31.44) is a continuous metric with a jump discontinuity of the extrinsic curvature across the hypersurface of codimension one $\rho=\xi$. Then the metric (31, 44) is a regular metric $\forall \xi>0$. We have

$$
\mathbf{g}=\eta-(1-H)\left(\mathbf{d} \rho \otimes \mathbf{d} \rho+\rho^{2} \mathbf{d} \varphi \otimes \mathbf{d} \varphi\right)
$$

and

$$
\mathbf{g}^{-1}=\eta^{-1}-\left(1-H^{-1}\right)\left(\partial_{\rho} \otimes \partial_{\rho}+\rho^{-2} \partial_{\varphi} \otimes \partial_{\varphi}\right),
$$

where $\eta$ is given by (12), $H$ is given by (44) and we take for the differentiable structure that in which $t, x=\rho \cos \varphi$ and $y=\rho \sin \varphi$ form a smooth chart. Hence it follows that $\mathbf{g}$ and $\mathbf{g}^{-1}$ exist everywhere.

Let $\mathbf{U}$ be a test $\left(\begin{array}{l}2 \\ 0\end{array}\right)$-tensor field on $\mathcal{R}^{3}$. We have

$$
\mathbf{g}[\mathbf{U}]=\int_{\mathcal{R}^{3}}(\eta \mid \mathbf{U}) \omega_{\eta}-\int d t \int_{0}^{\xi} d \rho \int d \varphi \rho\left(1-\xi^{-8 m}\right)\left(U^{x x}+U^{y y}\right)-\int d t \int_{\xi}^{\infty} d \rho \int d \varphi \rho\left(1-\rho^{-8 m}\right)\left(U^{x x}+U^{y y}\right),
$$

where $\rho=\sqrt{x^{2}+y^{2}}$. Therefore, $\mathbf{g}$ is locally bounded for $\xi>0$. Next, let $\mathbf{S}$ be a test $\left(\begin{array}{c}0 \\ 2\end{array}\right)$-tensor field on $\mathcal{R}^{3}$. It follows that

$$
\mathbf{g}^{-1}[\mathbf{S}]=\int_{\mathcal{R}^{3}}\left(\mathbf{S} \mid \eta^{-1}\right) \omega_{\eta}-\int d t \int_{0}^{\xi} d \rho \int d \varphi \rho\left(1-\xi^{8 m}\right)\left(S_{x x}+S_{y y}\right)-\int d t \int_{\xi}^{\infty} d \rho \int d \varphi \rho\left(1-\rho^{8 m}\right)\left(S_{x x}+S_{y y}\right) .
$$


Hence, $\mathbf{g}^{-1}$ is locally bounded for all $\xi>0$. Finally, let $\mathbf{U}$ be a test $\left(\begin{array}{l}3 \\ 0\end{array}\right)$-tensor field on $\mathcal{R}^{3}$. We find that the weak derivative in $\eta$ of $\mathbf{g}$ is given by

$$
\nabla \mathbf{g}[\mathbf{U}]=-\mathbf{g}[\eta \cdot \nabla \mathbf{U}]=\int_{\mathcal{R}^{3}} W_{c a b} U^{c a b} \omega_{\eta}
$$

where

$$
W_{c a b}= \begin{cases}0, & \rho<\xi \\ -8 m \rho^{-(1+8 m)} d \rho_{c}\left(d \rho_{a} d \rho_{b}+\rho^{2} d \varphi_{a} d \varphi_{b}\right), & \rho>\xi\end{cases}
$$

It then follows that $\nabla \mathbf{g}$ is locally square integrable for $\xi>0$. Therefore, (31, 44 ) is a regular metric. Furthermore, since it is a continuous metric, we have a well defined intrinsic 2-geometry at the two-surface $\rho=\xi$. It should be recalled that continuous regular metrics can be suitably approximated by smooth metrics [4]. In this sense, the class of continuous regular metrics provides a physically sensible idealization of the smooth metrics of general relativity.

Now, from (31, 44) and (9) it follows that

$$
C_{a b}^{c}= \begin{cases}0, & \rho<\xi \\ \frac{4 m}{\rho}\left(\partial_{\rho}^{c} d \rho_{a} d \rho_{b}+\partial_{\varphi}^{c}\left(d \rho_{a} d \varphi_{b}+d \rho_{b} d \varphi_{a}\right)-\rho^{2} \partial_{\rho}^{c} d \varphi_{a} d \varphi_{b}\right), & \rho>\xi\end{cases}
$$

and

$$
C_{m[b}^{d} C_{a] c}^{m}=0
$$

From (8), (55) and (56) we have

$$
R_{a b}=\nabla_{[c} C_{a] b}^{c} .
$$

Computations analogous to the previous ones give

$$
R_{a b}\left[S^{a b}\right]=4 m \int d t \int d \varphi\left(S^{x x}(t, \xi \cos \varphi, \xi \sin \varphi)+S^{y y}(t, \xi \cos \varphi, \xi \sin \varphi)\right)
$$

and

$$
\begin{aligned}
G_{a b}\left[S^{a b}\right]= & R_{a b}\left[S^{a b}\right] \\
& -4 m \int d t \int d \varphi\left(-\xi^{8 m} S^{t t}(t, \xi \cos \varphi, \xi \sin \varphi)+S^{x x}(t, \xi \cos \varphi, \xi \sin \varphi)+S^{y y}(t, \xi \cos \varphi, \xi \sin \varphi)\right) \\
= & 4 m \xi^{8 m} \int d t \int d \varphi S^{t t}(t, \xi \cos \varphi, \xi \sin \varphi),
\end{aligned}
$$

where in the last step we have used (58). Hence

$$
\lim _{\xi \rightarrow 0} R_{a b}\left[S^{a b}\right]=8 \pi m \delta_{(0)}^{(2)}\left(d x_{a} d x_{b}+d y_{a} d y_{b}\right)\left[S^{a b}\right]
$$

and

$$
\lim _{\xi \rightarrow 0} G_{a b}\left[S^{a b}\right]=0
$$

in agreement with (26) and (30).

For the sake of comparison, let us calculate $G_{b}^{a}$ for the metric (31,44). For a smooth metric we have

$$
G_{b}^{a}=R_{b}^{a}-\frac{1}{2}\left(g^{-1}\right)^{c d} \tilde{R}_{c d} \delta_{b}^{a}+\left(g^{-1}\right)^{c d} C_{m[c}^{e} C_{e] d}^{m} \delta_{b}^{a}+\nabla_{[c}\left(C_{e] d}^{e}\left(g^{-1}\right)^{c d}\right) \delta_{b}^{a}+C_{d[c}^{e} \nabla_{e]}\left(g^{-1}\right)^{c d} \delta_{b}^{a},
$$

where

$$
R_{b}^{a}=\left(g^{-1}\right)^{a c} \tilde{R}_{c b}+2 \nabla_{[c}\left(C_{d] b}^{c}\left(g^{-1}\right)^{a d}\right)+2 C_{b[c}^{c} \nabla_{d]}\left(g^{-1}\right)^{a d}+2\left(g^{-1}\right)^{a d} C_{m[c}^{c} C_{d] b}^{m}
$$


We take (62) as the definition of the mixed Einstein tensor for a regular metric. As a qualifying remark, it should be recalled that for a regular metric, the outer product of any number of metrics and inverse metrics with a single curvature tensor can be interpreted as a distribution [4]. Since any contraction of a distribution is a distribution, for a regular metric the Einstein tensors (10) and (62) are well defined as distributions.

Let $\mathbf{S}$ be a test $\left(\begin{array}{l}1 \\ 1\end{array}\right)$-tensor field on $\mathcal{R}^{3}$. From (62) and (63), a calculation analogous to that of equation (25) leads to

$$
R_{b}^{a}\left[S_{a}^{b}\right]=4 m \xi^{8 m} \int d t \int d \varphi\left(S_{x}^{x}(t, \xi \cos \varphi, \xi \sin \varphi)+S_{y}^{y}(t, \xi \cos \varphi, \xi \sin \varphi)\right)
$$

and

$$
\begin{aligned}
G_{b}^{a}\left[S_{a}^{b}\right] & =R_{b}^{a}\left[S_{a}^{b}\right]-4 m \xi^{8 m} \int d t \int d \varphi\left(S_{t}^{t}(t, \xi \cos \varphi, \xi \sin \varphi)+S_{x}^{x}(t, \xi \cos \varphi, \xi \sin \varphi)+S_{y}^{y}(t, \xi \cos \varphi, \xi \sin \varphi)\right) \\
& =-4 m \xi^{8 m} \int d t \int d \varphi S_{t}^{t}(t, \xi \cos \varphi, \xi \sin \varphi),
\end{aligned}
$$

where we have used (64). Hence

$$
\lim _{\xi \rightarrow 0} R_{b}^{a}\left[S_{a}^{b}\right]=0
$$

and

$$
\lim _{\xi \rightarrow 0} G_{b}^{a}\left[S_{a}^{b}\right]=0 .
$$

A comparison of (47) and (67) illustrates the dependence of (67) on the volume element chosen. This is the usual situation when tensors distributions are associated with locally integrable tensors via Eq.(3), requiring the specification of a reference volume element [14]. However, as this example suggests, the approach that led us to (47) appears to be more appropriate than the one used to arrive to (30) and (67), in order to relate the singularity of the curvature of (11) to that of the Einstein tensor.

Finally, since general relativity is a covariant theory, coordinate invariance of these results must be considered. The present approach needs the introduction of a coordinate system but, since the righthand side of (47) is a tensor density of weight one defined on $\mathcal{R}^{2}$, coordinate invariance of the result is expected at least under those coordinate transformations that do not involve the coordinate $t$.

\section{THE SCHWARZSCHILD GEOMETRY}

The Schwarzschild solution is locally the only asymptotically flat, spherically symmetric solution to the Einstein empty space field equations. Schwarzschild spacetime is taken usually as that part of a 4-dimensional manifold with a metric of the form

$$
\mathbf{g}=-\left(1-\frac{2 m}{r}\right) \mathbf{d} t \mathbf{d} t+\left(1-\frac{2 m}{r}\right)^{-1} \mathbf{d} r \mathbf{d} r+r^{2}\left(\mathbf{d} \theta \mathbf{d} \theta+\sin ^{2} \theta \mathbf{d} \varphi \mathbf{d} \varphi\right) .
$$

where $r>2 m,-\infty<t<\infty, 0 \leq \theta \leq \pi$ and $0 \leq \varphi \leq 2 \pi$. Following standard practice, in (68) we have omitted writing the outer product sign.

Assuming that the manifold and (68) can be extended to include the region $r \leq 2 m$, we can ask for the source of this geometry. The metric (68) does not fall within the class of regular metrics 沺. In the following, we will show that it is not a semi-regular metric.

Let $\eta$ be the ordinary Minkowski metric on $\mathcal{R}^{4}$ given by

$$
\eta=-\mathbf{d} t \mathbf{d} t+\mathbf{d} r \mathbf{d} r+r^{2}\left(\mathbf{d} \theta \mathbf{d} \theta+\sin ^{2} \theta \mathbf{d} \varphi \mathbf{d} \varphi\right) .
$$

We take for the differentiable structure that in which $t, x=r \cos \varphi \sin \theta, y=r \sin \varphi \sin \theta$ and $z=r \cos \theta$ form a smooth chart. It follows that

$$
\mathbf{g}=\eta+\frac{2 m}{r} \mathbf{d} t \mathbf{d} t-\frac{2 m}{2 m-r} \mathbf{d} r \mathbf{d} r
$$


and

$$
\mathbf{g}^{-1}=\eta^{-1}+\frac{2 m}{2 m-r} \partial_{t} \partial_{t}-\frac{2 m}{r} \partial_{r} \partial_{r}
$$

where $r=\sqrt{x^{2}+y^{2}+z^{2}}$. We have that $\mathbf{g}$ and $\mathbf{g}^{-1}$ exist almost everywhere. Let $\mathbf{U}$ be a test $\left(\begin{array}{l}2 \\ 0\end{array}\right)$-tensor field on $\mathcal{R}^{4}$ with support on $r<2 m$. Thus,

$$
\mathbf{g}[\mathbf{U}]=\int_{\mathcal{R}^{4}}(\eta \mid \mathbf{U}) \omega_{\eta}-\int_{\mathcal{R}^{3}}\left(\frac{2 m}{r} U^{t t}-\frac{2 m}{2 m-r} d r_{a} d r_{b} U^{a b}\right) \omega_{\eta} .
$$

Therefore, $\mathbf{g}$ is locally integrable. Now, let $\mathbf{S}$ be a test $\left(\begin{array}{l}0 \\ 2\end{array}\right)$-tensor field on $\mathcal{R}^{4}$ with support on $r<2 m$. It follows that

$$
\mathbf{g}^{-1}[\mathbf{S}]=\int_{\mathcal{R}^{4}}\left(\mathbf{S} \mid \eta^{-1}\right) \omega_{\eta}-\int_{\mathcal{R}^{4}}\left(\frac{2 m}{2 m-r} S_{t t}-\frac{2 m}{r} \partial_{r}^{a} \partial_{r}^{b} S_{a b}\right) \omega_{\eta}
$$

Hence, $\mathbf{g}^{-1}$ is locally integrable. Next, let $\mathbf{U}$ be a test $\left(\begin{array}{l}3 \\ 0\end{array}\right)$-tensor field on $\mathcal{R}^{4}$ with support on $r<2 m$. The weak derivative in $\eta$ of $\mathbf{g}$ exist almost everywhere and is given by

$$
\nabla \mathbf{g}[\mathbf{U}]=-\mathbf{g}[\eta \cdot \nabla \mathbf{U}]=\lim _{\epsilon \rightarrow 0} \int_{r>\epsilon} g_{a b} \nabla_{c} U^{c a b} \omega_{\eta}=\int_{\mathcal{R}^{4}} W_{c a b} U^{c a b} \omega_{\eta}
$$

where

$$
\begin{aligned}
W_{c a b}= & -\frac{2 m}{r^{2}} d r_{c} d t_{a} d t_{b}-\frac{2 m}{(24-r)^{2}} d r_{c} d r_{a} d r_{b} \\
& -\frac{1}{r} \frac{2 m}{2 m-r}\left(r^{2}\left(d \theta_{c} d \theta_{a}+\sin ^{2} \theta d \varphi_{c} d \varphi_{a}\right) d r_{b}+r^{2} d r_{a}\left(d \theta_{c} d \theta_{b}+\sin ^{2} \theta d \varphi_{c} d \varphi_{b}\right)\right) .
\end{aligned}
$$

It then follows that $\nabla \mathbf{g}$ is locally integrable. However, it is not locally square-integrable in $r<2 m$ due to the fact that $-\frac{2 m}{r^{2}} d r_{c} d t_{a} d t_{b}$ is not locally square-integrable. Finally, from (9) and (75) we have

$$
\begin{aligned}
C_{a b}^{c}= & -\frac{1}{r} \frac{m}{2 m-r} \partial_{t}^{c}\left(d r_{a} d t_{b}+d t_{a} d r_{b}\right)+\frac{1}{r} \frac{m}{2 m-r} \partial_{r}^{c} d r_{a} d r_{b} \\
& +\frac{2 m}{r^{2}} \partial_{r}^{c}\left(r^{2} d \theta_{a} d \theta_{b}+r^{2} \sin ^{2} \theta d \varphi_{a} d \varphi_{b}\right)-\left(\frac{2 m}{r^{3}}-\frac{m}{r^{2}}\right) \partial_{r}^{c} d t_{a} d t_{b},
\end{aligned}
$$

which is not locally integrable because $\frac{2 m}{r^{3}} \partial_{r}^{c} d t_{a} d t_{b}$ is not locally integrable. Therefore the metric (68) is not a semi-regular metric.

In these case, however, distributional curvature and Einstein tensors can be obtained for (68) through regularization procedures. Following Ref. [15], consider a metric of the form

$$
\mathbf{g}=h \mathbf{d} t \mathbf{d} t-h^{-1} \mathbf{d} r \mathbf{d} r+r^{2}\left(\mathbf{d} \theta \mathbf{d} \theta+\sin ^{2} \theta \mathbf{d} \varphi \mathbf{d} \varphi\right),
$$

where $0 \leq r<2 m$ and $h$ is a $C^{\infty}$ function of $r$. The evaluation of the Einstein tensor proceeds now in a straightforward manner. We omit the details of the calculation; the result is

$$
G=-\left[\frac{1}{r^{2}} \frac{d}{d r}(r(1+h))\right]\left(\mathbf{d} t \partial_{t}+\mathbf{d} r \partial_{r}\right)-\frac{1}{2}\left[\frac{1}{r^{2}} \frac{d}{d r}\left(r^{2} \frac{d}{d r} h\right)\right]\left(\mathbf{d} \theta \partial_{\theta}+\mathbf{d} \varphi \partial_{\varphi}\right) .
$$

To find a distributional source for the Schwarzschild spacetime, consider the components of (78) as distributions on the space of test functions $\phi \in \mathcal{D}\left(\mathcal{R}^{3}\right)$ with support on the region $r<2 m$, where $\mathcal{R}^{3}$ is endowed with the usual $C^{\infty}$ Euclidean metric, and $h$ given by

$$
h(r)=-1+\frac{2 m}{r} f,
$$

where $f=f_{\lambda}(r)$ is a $C^{\infty}$ function such that $f_{\lambda}(0)=0$ and satisfying $\lim _{\lambda \rightarrow \lambda_{0}} f_{\lambda}(r) \rightarrow 1$. The metric (77, 89$)$ is a regularized version of (68). From Einstein field equations and (78.79), a source $\mathbf{T}_{f}$ which depends explicitly on the regularization function $f_{\lambda}$ is obtained. By taking $f_{\lambda}(r)=r^{\lambda}$, the $\lambda \rightarrow 0$ distributional limit, gives 


$$
\mathbf{T}=-m \delta_{(0)}^{(3)}\left[\mathbf{d} t \partial_{t}+\mathbf{d} r \partial_{r}-\frac{1}{2} \mathbf{d} \theta \partial_{\theta}-\frac{1}{2} \mathbf{d} \varphi \partial_{\varphi}\right]
$$

where $\delta_{(0)}^{(3)}$ is the usual three-dimensional Euclidean $\delta$ distribution. By virtue of the distributional identity in $\mathcal{R}^{3}$

$$
\delta_{(0)}^{(3)}\left[\mathbf{d} r \partial_{r}-\frac{1}{2} \mathbf{d} \theta \partial_{\theta}-\frac{1}{2} \mathbf{d} \varphi \partial_{\varphi}\right]=0
$$

it follows that (80) has the simpler expression

$$
\mathbf{T}=-m \delta_{(0)}^{(3)} \mathbf{d} t \partial_{t}
$$

The distributional tensor $\mathbf{T}$ given by (82), vanishes for $m=0$ and has support on the region $|\vec{x}|=0$. Therefore, the Schwarzschild geometry can be considered as generated by the distributional energy-momentum tensor (82) 15]. It should be noted that (32) can also be obtained without the need of regularization procedures, approximating (68) by analytic metrics and using the fact that reciprocals of analytic functions provide well-defined distributions [1].

We shall now show that the regularization (79) with $f_{\lambda}(r)=r^{\lambda}$ does not provide a regular metric in the sense of reference 断. Let $\mathbf{U}$ and $\mathbf{S}$ be a test $\left(\begin{array}{l}2 \\ 0\end{array}\right)$-tensor and $\left(\begin{array}{l}0 \\ 2\end{array}\right)$-tensor fields on $\mathcal{R}^{4}$, respectively, with support on $r<2 m$. We have

$$
(\mathbf{g} \mid \mathbf{U})=(\eta \mid \mathbf{U})-\left(\frac{2 m r^{\lambda}}{r} U^{t t}-\frac{2 m r^{\lambda}}{2 m r^{\lambda}-r} d r_{a} d r_{b} U^{a b}\right)
$$

and

$$
\left(\mathbf{g}^{-1} \mid \mathbf{S}\right)=\left(\mathbf{S} \mid \eta^{-1}\right)-\left(\frac{2 m r^{\lambda}}{2 m r^{\lambda}-r} S_{t t}-\frac{2 m r^{\lambda}}{r} \partial_{r}^{a} \partial_{r}^{b} S_{a b}\right) .
$$

It then follows that $\mathbf{g}$ and $\mathbf{g}^{-1}$, although locally integrable for $\lambda \geq 0$, are locally bounded for $\lambda \geq 1$. Further, the weak derivative in $\eta$ of $\mathbf{g}$ exist almost everywhere and is given by

$$
\nabla \mathbf{g}[\mathbf{U}]=-\mathbf{g}[\eta \cdot \nabla \mathbf{U}]=\int_{\mathcal{R}^{4}} W_{c a b} U^{c a b} \omega_{\eta}
$$

where

$$
W_{c a b}=-(1-\lambda) 2 m r^{\lambda-2} d r_{c} d t_{a} d t_{b}-2 m \nabla_{c}\left(\frac{r^{\lambda}}{2 m r^{\lambda}-r} d r_{a} d r_{b}\right)
$$

The weak derivative is locally integrable for $\lambda \geq 0$ and locally square integrable for $\lambda \geq \frac{1}{2}$. It then follows that this regularization does not provide a regular metric $\forall \lambda>0$. Thus the distributional meaning of the Riemann tensor and its contractions, in the sense of reference 顿, is questionable at the intermediate steps of the calculation.

We note in passing that (82) may be obtained with different regularization functions $f_{\lambda}$ 15. 16. However, the invariance of the regularization procedure is uncertain and (82) is obtained only by imposing some rather ad hoc regularization prescriptions [16]. Using the Kerr-Schild ansatz for the metric tensor and assuming that under the regularization procedure the metric maintains its Kerr-Schild form, it has been shown that (82) can also be obtained [17.

We turn now to the problem posed by Einstein field equations $\mathbf{G}=8 \pi \mathbf{T}$, with $\mathbf{T}$ given by (82). What we want to address here is how to arrive to (82) by a regularization procedure guaranteeing that the curvature tensor and its contractions be well defined tensor distributions at all intermediate steps of the calculation. The regularization procedure discussed in the previous section can be applied here. As we have shown, within this approach many of the difficulties arising from lack of invariance or caused by regularization ambiguities are avoided, or at least, deferred.

We take $(78)$ as a definition of the Einstein tensor. Let $\mathbf{U}$ be a test $\left(\begin{array}{l}1 \\ 1\end{array}\right)$-tensor field on $\mathcal{R}^{4}$. Next, consider the mixed-index Einstein tensor density $|\operatorname{det} \mathbf{g}|^{\frac{1}{2}} \mathbf{G}$, with $|\operatorname{det} \mathbf{g}|^{\frac{1}{2}}$ the density generated by the metric $\mathbf{g}$ in $(77)$, as a functional on the space of test tensor fields through

$$
\int_{\mathcal{R}^{4}}|\operatorname{det} \mathbf{g}|^{\frac{1}{2}} G_{b}^{a} U_{a}^{b} \varepsilon=\int_{\mathcal{R}^{4}} d t d x d y d z G_{b}^{a} U_{a}^{b},
$$


where it is understood that $G_{b}^{a}$ and $U_{a}^{b}$ are Cartesian components as functions of Cartesian coordinates $(t, x, y, z)$ with $x=r \cos \varphi \sin \theta, y=r \sin \varphi \sin \theta$ and $z=r \cos \theta$. Then

$$
\begin{aligned}
\int_{\mathcal{R}^{4}}|\operatorname{det} \mathbf{g}|^{\frac{1}{2}} G_{b}^{a} U_{a}^{b} \varepsilon=\lim _{\epsilon \rightarrow 0} 4 \pi \int_{r>\epsilon} d r & {\left[-\frac{d}{d r}(r(1+h))\right]\left(\overline{U_{t}^{t}}(r)+\overline{\partial_{r}^{a} d r_{b} U_{a}^{b}}(r)\right) } \\
+ & \frac{1}{2}\left[-\frac{d}{d r}\left(r^{2} \frac{d}{d r} h\right)\right] \overline{\left(\partial_{\theta}^{a} d \theta_{b}+\partial_{\varphi}^{a} d \varphi_{b}\right) U_{a}^{b}}(r),
\end{aligned}
$$

where

$$
\begin{aligned}
& \overline{U_{t}^{t}}(r) \equiv \frac{1}{4 \pi} \int_{-\infty}^{\infty} d t \int_{0}^{\pi} d \theta \int_{0}^{2 \pi} d \varphi \sin \theta U_{t}^{t} \\
& \overline{\partial_{r}^{a} d r_{b} U_{a}^{b}}(r) \equiv \frac{1}{4 \pi} \int_{-\infty}^{\infty} d t \int_{0}^{\pi} d \theta \int_{0}^{2 \pi} d \varphi \sin \theta {\left[\sin ^{2} \theta\left(\cos ^{2} \varphi U_{x}^{x}+\sin ^{2} \varphi U_{y}^{y}\right)+\cos ^{2} \theta U_{z}^{z}\right.} \\
&+\sin ^{2} \theta \sin \varphi \cos \varphi\left(U_{x}^{y}+U_{y}^{x}\right) \\
&+\sin \theta \cos \theta \sin \varphi\left(U_{z}^{y}+U_{y}^{z}\right) \\
&\left.+\sin \theta \cos \theta \cos \varphi\left(U_{x}^{z}+U_{z}^{x}\right)\right]
\end{aligned}
$$

and

$$
\begin{aligned}
\overline{\left(\partial_{\theta}^{a} d \theta_{b}+\partial_{\varphi}^{a} d \varphi_{b}\right) U_{a}^{b}}(r) \equiv \frac{1}{4 \pi} \int_{-\infty}^{\infty} d t \int_{0}^{\pi} d \theta \int_{0}^{2 \pi} d \varphi \sin \theta[ & \cos ^{2} \theta\left(\cos ^{2} \varphi U_{x}^{x}+\sin ^{2} \varphi U_{y}^{y}\right)+\sin ^{2} \theta U_{z}^{z}+ \\
& +\sin ^{2} \varphi U_{x}^{x}+\cos ^{2} \varphi U_{y}^{y} \\
& -\sin ^{2} \theta \sin \varphi \cos \varphi\left(U_{x}^{y}+U_{y}^{x}\right) \\
& -\sin \theta \cos \theta \sin \varphi\left(U_{z}^{y}+U_{y}^{z}\right) \\
& \left.-\sin \theta \cos \theta \cos \varphi\left(U_{x}^{z}+U_{z}^{x}\right)\right]
\end{aligned}
$$

It follows

$$
\int_{\mathcal{R}^{4}}|\operatorname{det} \mathbf{g}|^{\frac{1}{2}} G_{b}^{a} U_{a}^{b} \varepsilon=\lim _{\epsilon \rightarrow 0}\left\{4 \pi \int_{\epsilon}^{\infty} d r r(1+h) \frac{d}{d r}\left[\overline{U_{t}^{t}}+\overline{\partial_{r}^{a} d r_{b} U_{a}^{b}}\right]-2 \pi \int_{\epsilon}^{\infty} h \frac{d}{d r}\left[r^{2} \frac{d}{d r} \overline{\left(\partial_{\theta}^{a} \theta_{b}+\partial_{\varphi}^{a} d \varphi_{b}\right) U_{a}^{b}}\right]\right\},
$$

where we have integrated by parts an imposed the condition

$$
\lim _{r \rightarrow 0} r^{2} \frac{d}{d r} h=0 .
$$

Now, let us assume that (92) holds even for a less well-behaved $h$. Let $h$ be the $C^{1}$-function given by

$$
h=-1+\frac{2 m}{r_{>}},
$$

where $r_{>}=\max \{r, \xi\}$ with $0<\xi<2 m$ a constant parameter which we will treat as a regulator. Eq. 94 is the solution of

$$
\frac{d}{d r}\left(r^{2} \frac{d}{d r} h\right)=-2 m \delta(\rho-\xi),
$$

where both sides should be understood as distributions on test functions $\phi(r) \in \Phi\left(\mathcal{R}^{+}\right)$whose support is contained in $r<2 m$. Further, (94) satisfies (93) and

$$
\left.h\right|_{r=2 m}=0,
$$

where the condition (96) ensures continuity of the metric tensor at $r=2 m$, assuming that (68) holds for $r>2 m$. Note that, although for $r<2 m$ the coordinate $r$ is timelike and the spacetime is no static, causality with respect to $r$ cannot be used as a criterion to obtain $h$.

From (92) and (94) it follows 


$$
\int_{\mathcal{R}^{4}}|\operatorname{det} \mathbf{g}|^{\frac{1}{2}} G_{b}^{a} U_{a}^{b} \varepsilon=-8 \pi m \frac{1}{\xi} \int_{0}^{\xi} d r\left[\overline{U_{t}^{t}}+\overline{\partial_{r}^{a} d r_{b} U_{a}^{b}}\right]+4 \pi m \overline{\left(\partial_{\theta}^{a} \theta_{b}+\partial_{\varphi}^{a} d \varphi_{b}\right) U_{a}^{b}}(\xi) .
$$

Eq. (97) implies that the density $|\operatorname{det}|^{\frac{1}{2}} \mathbf{G}$ has support on the four dimensional submanifold $r<\xi$.

Now, from (97) we have

$$
\lim _{\xi \rightarrow 0} \int_{\mathcal{R}^{4}}|\operatorname{det} \mathbf{g}|^{\frac{1}{2}} G_{b}^{a} U_{a}^{b} \varepsilon=-8 \pi m\left[\overline{U_{t}^{t}}(0)+\overline{\partial_{r}^{a} d r_{b} U_{a}^{b}}(0)\right]+4 \pi m \overline{\left(\partial_{\theta}^{a} \theta_{b}+\partial_{\varphi}^{a} d \varphi_{b}\right) U_{a}^{b}}(0),
$$

where

$$
\overline{\partial_{r}^{a} d r_{b} U_{a}^{b}}(0)=\frac{1}{3} \int_{-\infty}^{\infty} d t\left(U_{x}^{x}(t, 0,0,0)+U_{y}^{y}(t, 0,0,0)+U_{z}^{z}(t, 0,0,0)\right)
$$

and

$$
\overline{\left(\partial_{\theta}^{a} \theta_{b}+\partial_{\varphi}^{a} d \varphi_{b}\right) U_{a}^{b}}(0)=\frac{2}{3} \int_{-\infty}^{\infty} d t\left(U_{x}^{x}(t, 0,0,0)+U_{y}^{y}(t, 0,0,0)+U_{z}^{z}(t, 0,0,0)\right) .
$$

It follows

$$
\lim _{\xi \rightarrow 0} \int_{\mathcal{R}^{4}}|\operatorname{det} \mathbf{g}|^{\frac{1}{2}} G_{b}^{a} U_{a}^{b} \varepsilon=-8 \pi m \int_{-\infty}^{\infty} d t U_{t}^{t}(t, 0,0,0) .
$$

Therefore, the $\xi \rightarrow 0$ limit of the density $|\operatorname{det} \mathbf{g}|^{\frac{1}{2}} \mathbf{G}$ is the distribution

$$
\lim _{\xi \rightarrow 0}|\operatorname{det} \mathbf{g}|^{\frac{1}{2}} \mathbf{G}=-8 \pi m \delta_{(0)}^{(3)} \mathbf{d} t \partial t
$$

where $\delta_{(0)}^{(3)}$ is the usual three-dimensional Euclidean $\delta$ distribution with support on the origin. This means that the spacetime $\left(\mathcal{R}^{4}, \mathbf{g}\right)$, where the metric $\mathbf{g}$ is given by (77,94), can be identified in the $\xi \rightarrow 0$ limit with the spacetime generated by the distributional energy-momentum tensor $\mathbf{T}$ for which

$$
|\operatorname{det} \mathbf{g}|^{\frac{1}{2}} \mathbf{T}=-m \delta_{(0)}^{(3)} \mathbf{d} t \partial_{t} .
$$

Since for $r>\xi$, the metrics (68) and (77) with $h$ given by (94) coincide, a point source of mass $m$ at the origin may be considered as the source for the Schwarzschild geometry, as claimed in references [1].15. Note that since the righthand side of (102) is a tensor density of weight one defined on $\mathcal{R}^{3}$, coordinate invariance of this result is expected at least under those coordinate transformations that do not involve the coordinate $t$.

As follows from (94), $h$ is continuous at $r=\xi$. Therefore, the usually required continuity of the metric is ensured and the intrinsic 3-geometry of the hypersurface $r=\xi$ is well defined. On the other hand, the extrinsic curvature is discontinuous across this surface of codimension one. It then follows that the metric (77) 94 is a regular metric $\forall \xi>0$. We have

$$
\mathbf{g}=\eta+\frac{2 m}{r_{>}} \mathbf{d} t \mathbf{d} t-\frac{2 m}{2 m-r_{>}} \mathbf{d} r \mathbf{d} r
$$

and

$$
\mathrm{g}^{-1}=\eta^{-1}+\frac{2 m}{2 m-r_{>}} \partial_{t} \partial_{t}-\frac{2 m}{r_{>}} \partial_{r} \partial_{r}
$$

Thus $\mathbf{g}$ and $\mathbf{g}^{-1}$ exist almost everywhere and are locally bounded $\forall \xi>0$. Let $\mathbf{U}$ be a test $\left(\begin{array}{c}3 \\ 0\end{array}\right)$-tensor field on $\mathcal{R}^{4}$ with support on $r<2 m$. The weak derivative in $\eta$ of $\mathbf{g}$ exist almost everywhere and is given by

$$
\nabla \mathbf{g}[\mathbf{U}]=\int_{\mathcal{R}^{4}} W_{c a b} U^{c a b} \omega_{\eta}
$$

where 


$$
W_{c a b}= \begin{cases}-\frac{1}{r} \frac{2 m}{2 m-\xi}\left(r^{2}\left(d \theta_{c} d \theta_{a}+\sin ^{2} \theta d \varphi_{c} d \varphi_{a}\right) d r_{b}+r^{2} d r_{a}\left(d \theta_{c} d \theta_{b}+\sin ^{2} \theta d \varphi_{c} d \varphi_{b}\right)\right), & r<\xi \\ -\frac{2 m}{r^{2}} d r_{c} d t_{a} d t_{b}-\frac{2 m}{(24-r)^{2}} d r_{c} d r_{a} d r_{b} & \\ -\frac{1}{r} \frac{2 m}{2 m-r}\left(r^{2}\left(d \theta_{c} d \theta_{a}+\sin ^{2} \theta d \varphi_{c} d \varphi_{a}\right) d r_{b}+r^{2} d r_{a}\left(d \theta_{c} d \theta_{b}+\sin ^{2} \theta d \varphi_{c} d \varphi_{b}\right)\right), & r>\xi\end{cases}
$$

This weak derivative is locally square-integrable. Hence, (77,94) is a regular metric as expected.

Computations analogous to the previous ones show that

$$
\begin{aligned}
& R_{a b}\left[U^{a b}\right]=m \int_{r=\xi} \frac{1}{\xi^{2}}\left(\left(1-\frac{2 m}{\xi}\right) d t_{a} d t_{b}-\left(1-\frac{2 m}{\xi}\right)^{-1} d r_{a} d r_{b}\right) U^{a b} \sigma \\
& +\frac{2 m}{\xi} \int_{-\infty}^{\infty} d t \int_{0}^{\xi} d r \int_{0}^{\pi} d \theta \int_{0}^{2 \pi} d \varphi \sin \theta\left(r^{2} d \theta_{a} d \theta_{b}+r^{2} \sin ^{2} \theta d \varphi_{a} d \varphi_{b}\right) U^{a b}, \\
& G_{a b}\left[U^{a b}\right]=\frac{2 m}{\xi} \int_{-\infty}^{\infty} d t \int_{0}^{\xi} d r \int_{0}^{\pi} d \theta \int_{0}^{2 \pi} d \varphi \sin \theta\left(\left(1-\frac{2 m}{\xi}\right) d t_{a} d t_{b}-\left(1-\frac{2 m}{\xi}\right)^{-1} d r_{a} d r_{b}\right) U^{a b} \\
& -2 m \int_{r=\xi} \frac{1}{\xi^{2}}\left(r^{2} d \theta_{a} d \theta_{b}+r^{2} \sin ^{2} \theta d \varphi_{a} d \varphi_{b}\right) U^{a b} \sigma, \\
& R_{b}^{a}\left[U_{a}^{b}\right]=-\int_{r=\xi} \frac{1}{\xi^{2}}\left(\partial_{t}^{a} d t_{b}+\partial_{r}^{a} d r_{b}\right) U_{a}^{b} \sigma \\
& +\frac{2 m}{\xi} \int_{-\infty}^{\infty} d t \int_{0}^{\xi} d r \int_{0}^{\pi} d \theta \int_{0}^{2 \pi} d \varphi \sin \theta\left(\partial_{\theta}^{a} d \theta_{b}+\partial_{\varphi}^{a} d \varphi_{b}\right) U_{a}^{b}
\end{aligned}
$$

and

$$
\begin{aligned}
G_{b}^{a}\left[U_{a}^{b}\right]= & -\frac{2 m}{\xi} \int_{-\infty}^{\infty} d t \int_{0}^{\xi} d r \int_{0}^{\pi} d \theta \int_{0}^{2 \pi} d \varphi \sin \theta\left(\partial_{t}^{a} d t_{b}+\partial_{r}^{a} d r_{b}\right) U_{a}^{b} \\
& +m \int_{r=\xi} \frac{1}{\xi^{2}}\left(\partial_{\theta}^{a} d \theta_{b}+\partial_{\varphi}^{a} d \varphi_{b}\right) U_{a}^{b} \sigma
\end{aligned}
$$

where it is understood that $U^{a b}$ and $U_{b}^{a}$ are Cartesian components as functions of Cartesian coordinates.

From (108-111) it follows that we have well defined distributional Ricci and Einstein tensors $\forall \xi>0$. However, in the $\xi \rightarrow 0$ limit, only the mixed components of these tensors turn out to be well defined distributions,

$$
\lim _{\xi \rightarrow 0} R_{b}^{a}\left[U_{a}^{b}\right]=4 \pi m \delta_{(0)}^{(3)}\left[-\partial_{t}^{a} d t_{b}+\partial_{x}^{a} d x_{b}+\partial_{y}^{a} d y_{b}+\partial_{z}^{a} d z_{b}\right]\left[U_{a}^{b}\right]
$$

and

$$
\lim _{\xi \rightarrow 0} G_{b}^{a}\left[U_{a}^{b}\right]=-8 \pi m \delta_{(0)}^{(3)} \partial_{t}^{a} d t_{b}\left[U_{a}^{b}\right]
$$

Notice that the result (102) is recovered. In this case, both approaches give the same result since the volume element of the local auxiliary metric (69) agrees with the volume element of the regularized metric (77,94).

\section{CONCLUDING REMARKS}

We have proposed a particular kind of regularization for the metrics of two singular spacetimes: the 2+1-dimensional spacetime around a massive point source and the Schwarzschild spacetime. In these two rather different examples, we have shown that a satisfactory regularization procedure is obtained by requiring that (i) the $\operatorname{density}|\operatorname{det}|^{\frac{1}{2}} G_{b}^{a}$, associated to the Einstein tensor $G_{b}^{a}$ of the regularized metric, rather than the Einstein tensor itself, be a distribution and (ii) the regularized metric be a continuous metric with a discontinuous extrinsic curvature across a non-null hypersurface of codimension one. For these examples, the regularized metrics are regular metrics with well defined distributional curvature and Einstein tensors at all the intermediate steps of the calculation. The distributional limit, when the regularization is removed, assigns a well defined distribution to the mixed-index Einstein tensor density 
with support on the singularity. Further, some interesting relationships between the curvatures obtained in different approaches have been put forward. We have not discussed exhaustively the coordinate invariance of the results. This important issue will be considered elsewhere. But the strong constraints that the present approach imposes on the regularization procedure suggest that it may be useful to get further insight about the distributional meaning of the curvature of non-regular metrics.

[1] P. E. Parker, J. Math. Phys. 20 (1979) 1423.

[2] A. Taub, J. Math. Phys. 21 (1980) 1423.

[3] C. K. Raju, J. Phys. A: Math. Gen. 15 (1982) 1785.

[4] R. P. Geroch and J. Traschen, Phys. Rev. D 36 (1987) 1017.

[5] W. Israel, Nuovo Cimento 44 (1966) 1; ibid 48 (1967) 463.

[6] M. Khorrami and R. Mansouri, Phys. Rev 44 (1991) 557.

[7] M. Khorrami and R. Mansouri, J. Math. Phys. 35 (1994) 951.

[8] R. Mansouri and M. Khorrami, J. Math. Phys. 37 (1996) 5672.

[9] D. Garfinkle, Class. Quant. Grav. 16 (1999) 4101.

[10] J. Colombeau, Journal of Mathematical Analysis and Applications 94 (1983) 96; Bulletin of the American Mathematical Society 23 (1990) 251.

[11] C. J. S. Clarke, J. A. Vickers and J. P. Wilson Class. Quantum Grav. 13 (1996) 2485.

[12] J. P. Wilson Class. Quantum Grav. 14 (1997) 3337.

[13] J. A. Vickers and J. P. Wilson Class. Quantum Grav. 16 (1999) 579.

[14] J. Louko and R. D. Sorkin, Class. Quantum Grav. 14 (1997) 179.

[15] H. Balasin and H. Nachbagauer, Class. Quantum Grav. 10 (1993) 2271.

[16] T. Kawai and E. Sakane, Prog. Theor. Phys. 98 (1997) 69.

[17] H. Balasin and H. Nachbagauer, Class. Quantum Grav. 11 (1994) 1453.

[18] R. Arnowitt, S. Deser and C. W. Misner, Phys. Rev. Lett. 4 (1960) 375; Physical Review 120 (1960) 321.

[19] R. Arnowitt, S. Deser and C. W. Misner, Ann. Phys. (N.Y.) 33 (1965) 88.

[20] S. Deser, R. Jackiw and G. t'Hooft, Ann. Phys. (N.Y.) 152 (1984) 220.

[21] Y. Choquet-Bruhat and C. DeWitt-Morette with M. Dillard-Bleick, Analysis, Manifolds and Physics. Part I: Basics. (North-Holland, 1991).

[22] A. Lichnerowicz, Propagateurs, Commutateurs et Anticommutateurs en Relativité Générale, Publication I.H.E.S. No. 10 (1961).

[23] T. Dray, Int. J. Mod. Phys. D6 (1997) 717.

[24] G. de Rham, Differentiable Manifolds (Springer Verlag, 1984).

[25] S. Deser and R. Jackiw, Ann.Phys. (N.Y.) 192 (1989) 352.

[26] G. Grignani and C. Lee, Ann.Phys. (N.Y.) 196 (1989) 386. 